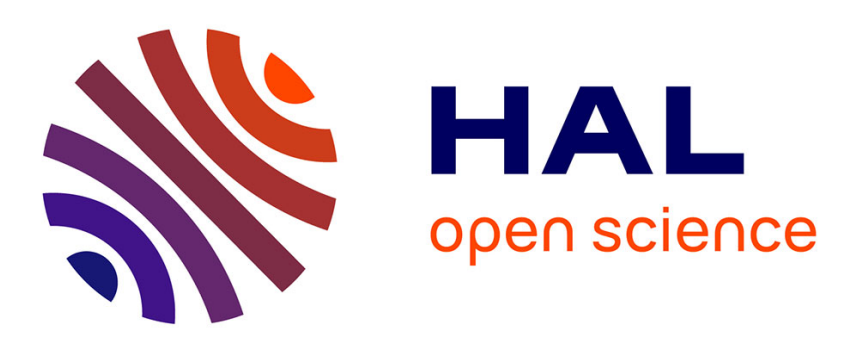

\title{
A Fourier-based numerical homogenization tool for an explosive material
}

\author{
Jean-Baptiste Gasnier, François Willot, Hervé Trumel, Bruno Figliuzzi, \\ Dominique Jeulin, Maxime Biessy
}

\section{> To cite this version:}

Jean-Baptiste Gasnier, François Willot, Hervé Trumel, Bruno Figliuzzi, Dominique Jeulin, et al.. A Fourier-based numerical homogenization tool for an explosive material. Matériaux \& Techniques, 2015, 103 (3), pp.308. 10.1051/mattech/2015019 . hal-01141495v2

HAL Id: hal-01141495

https://hal-mines-paristech.archives-ouvertes.fr/hal-01141495v2

Submitted on 19 May 2015

HAL is a multi-disciplinary open access archive for the deposit and dissemination of scientific research documents, whether they are published or not. The documents may come from teaching and research institutions in France or abroad, or from public or private research centers.
L'archive ouverte pluridisciplinaire HAL, est destinée au dépôt et à la diffusion de documents scientifiques de niveau recherche, publiés ou non, émanant des établissements d'enseignement et de recherche français ou étrangers, des laboratoires publics ou privés. 


\title{
A Fourier-based numerical homogenization tool for an explosive material
}

\author{
Jean-Baptiste Gasnier ${ }^{1}$, François Willot ${ }^{1}$, Hervé Trumel ${ }^{* 2}$, \\ Bruno Figliuzzi ${ }^{1}$, Dominique Jeulin ${ }^{1}$, and Maxime Biessy ${ }^{2}$ \\ ${ }^{1}$ MINES ParisTech, PSL - Research University, CMM - Centre for Mathematical Morphology ， \\ 35, rue St Honoré, F-77300 FONTAINEBLEAU, France \\ ${ }^{2}$ CEA, DAM, Le Ripault, F-37260 MONTS, France
}

\begin{abstract}
This paper describes the development of a numerical homogenization tool adapted to TATB-based pressed explosives. This is done by combining virtual microstructure modeling and Fourier-based computations. The polycrystalline microstructure is represented by a Johnson-Mehl tessellation model with Poisson random nucleation and anisotropic growth of grains. Several calculations are performed with several sets of available data for the thermoelastic behavior of TATB. Good agreement is found between numerical predictions and experimental data regarding the overall thermal expansion coefficient. The results are shown to comply with available bounds on polycrystalline anisotropic thermoelasticity. Finally, the size of the representative volume element is derived for the bulk, shear and volumetric thermal expansion moduli.
\end{abstract}

\section{INTRODUCTION}

W Ithin the wide class of civil or military energetic materials, pressed TATB-based explosives occupy a special place. TATB $(1,3,5-$ triamino-2,4,6-trinitrobenzene) is an energetic molecular crystal combining high energetic performance and very good thermal stability. By adding a small amount of a polymer acting as a binder between TATB grains, it is possible to obtain exceptionally safe explosives displaying good mechanical properties. These explosives exhibit a rate dependent quasi-brittle, concrete-like thermomechanical behavior [1, 2]. They are almost brittle under uniaxial tension, but undergo progressive damage by microcracking under uniaxial compression. As a result, the ratio between compressive and tensile fracture stress is roughly comprised between 5 and 10. They also display so-called "ratchet growth", i.e. irreversible dilatancy, under purely thermal loading [3, 4, 5]. This phenomenon is believed to be linked

\footnotetext{
${ }^{*}$ corresponding author
}

to the strongly anisotropic behavior of the TATB and to the random orientation of TATB grains in the macroscopically isotropic explosive [6].

Hence, the irreversibility of the macroscopic thermomechanical behavior of TATB-based plastic-bonded pressed explosives (PBXs) finds its sources at the microstructural level, referred to as the mesoscopic scale in the sequel. It is the authors' belief that significant progress in understanding and modeling such a behavior should involve mesoscopic numerical simulations. The development of a numerical thermomechanical tool operating at the level of the microstructure was therefore undertaken for a particular TATB based PBX. This tool uses virtual microstructure modeling and a Fourier-based numerical method.

The task is not simple, due to the complexity of the microstructure, the multiple sources of irreversibility, and the very limited available knowledge about the behavior of constituents and interfaces. The feasability 
of such a development was demonstrated in previous reports [7, 8], in a simplified context. The next steps will consist in incorporating progressively the various mechanisms responsible for non linear and irreversible response, mainly microcracking, plasticity and possibly viscoelasticity. Most of them being threshold phenomena, should be activated in zones of maximum load. Therefore, accurate predictions of the time and location of plastic yielding or grain-binder debonding can only be achieved within a realistic description of the microstructure. This necessary step is the main objective of this paper, which focuses on a more realistic, though still simplified, treatment of the microstructure, performed in this preparatory phase in the context of linear thermoelasticity.

Section III presents the material, its microstructure and macroscopic properties, the focus being put on its nonlinear thermal expansion. Section III describes the development of the virtual microstructure model. Section IV] gives the thermoelastic properties of the TATB single crystal, as extracted from bibliography. The main computational results are then given, the emphasis being put on the concept of representative volume element (RVE). The main findings are summarized in Section $\mathrm{V}$, which gives some insights about future work.

\section{THE MATERIAL}

The initial TATB powder is first coated with a small amount (less than $5 \%$ in mass) of an amorphous polymer in a slurry process. After careful drying, the coated powder is pressed under vacuum in an oil bath under high pressure (several hundred $\mathrm{MPa}$ ) and moderate temperature.

\section{Thermal expansion experiments}

Under thermal cycling, and provided that temperature exceeds a certain threshold, the specific volume of the material increases after each cycle, and eventually stabilizes after a number of cycles that depends upon the nature of the binder (see [9] among others), hence the denomination of "ratchet growth". Pressure tends to inhibit [10] this time dependent [9] effect. Ratchet growth is generally investigated above room temperature, and the glass transition of the binder is known to play a strong role on the magnitude of dilatancy, and on the temperature above which it manifests itself (see for example [3]). It is nowadays considered that the strongly anisotropic thermal expansion of TATB crystals induces internal stresses in a randomly oriented polycrystal [3, 6, 11].

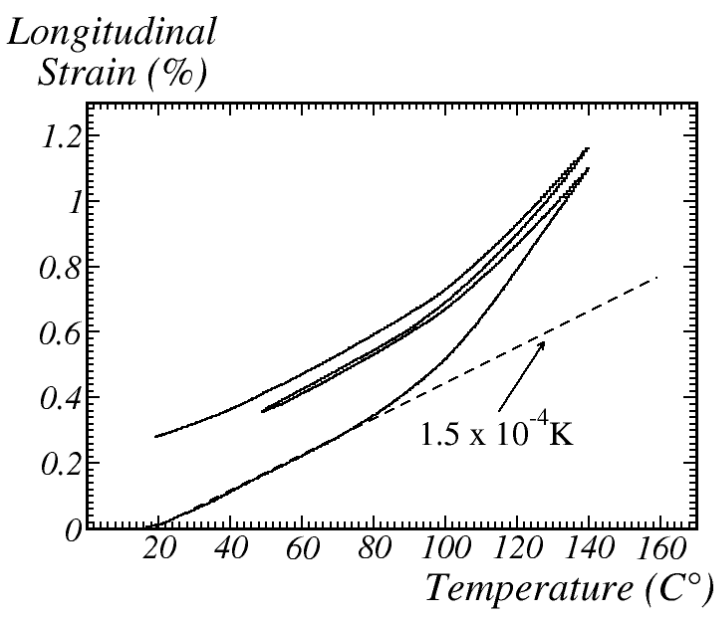

Figure 1: Linear expansion recorded during two thermal cycles above room temperature.

Fig. 1 shows the response of a $5 \times 5 \times 50 \mathrm{~mm}^{3}$ sample, at a heating/cooling rate of $\pm 10^{\circ} \mathrm{C}$ per hour. Each cycle is characterized by a linear response up to $70^{\circ} \mathrm{C}$ approximately. After a strongly nonlinear transient phase, a linear irreversible phase takes place, during which dilatancy occurs. Note that the dilatancy of the second cycle is much lower than that of the first one. Note also that the two cooling responses can be superposed, to a very good accuracy, except for the vertical shift corresponding to the dilatancy of the second cycle. The following interpretation is proposed for this response. From a free state of internal stresses at room temperature, internal stresses grow during the heating phase, especially in the binder. The latter softens progressively and relieves internal stresses, either by yielding or more likely by debonding locally. Upon cooling, opened microcracks tend to close partially, down to the glass transition, below which the binder 
re-hardens. Note that, according to this scenario, the free state of internal stresses is now at the re-hardening temperature of the binder.

If this scenario is correct, internal stresses should develop as well during cooling from room temperature. To verify this, a second thermal expansion experiment was performed at the same cooling/heating rate, but below room temperature. The sample (same geometry) was submitted to three thermal cycles down to 0 , -25 and $-50^{\circ} \mathrm{C}$ respectively. The result, displayed in Fig. 2. confirms the scenario. The first cycle is linear and strictly reversible. For the other two, a linear phase with the same thermal expansion coefficient as in Fig. 11 takes place down to a temperature threshold below which dilatancy occurs. Heating and re-cooling show that the thermal expansion coefficient has decreased, and decreases once more during the third cycle. This is a typical signature of a microcrack opening-closing process in response to the thermal strain. Note that upon returning to ambient temperature, the remaining dilatancy is much lower than in the case of Fig. 1. but that the coefficient of thermal expansion has varied irreversibly.

Therefore, the behavior of the binder appears as crucial. As internal stresses grow in response to heating or cooling, they are not relieved in the same way at high or low temperature. Above the glass transition, the very soft binder deforms easily, and relaxes internal strains by yielding, allowing grain reorganization and subsequent dilatancy. Below the glass transition, the very hard binder transmits internal stresses to grains, that are likely to fail, thus providing extra internal degrees of freedom and relaxing internal stresses by internal free surface development, with subsequent thermal expansion modulus variations, but little or no dilatancy.

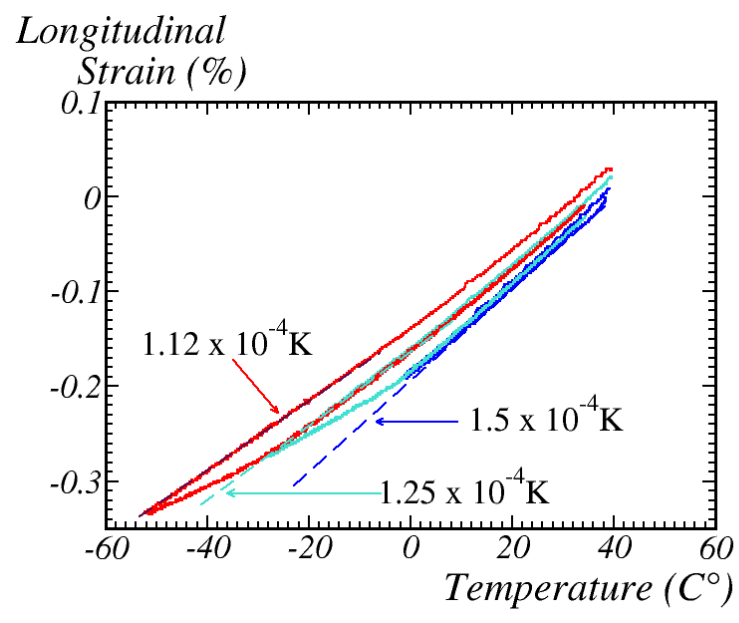

Figure 2: Linear expansion recorded during three thermal cycles below room temperature.

\section{Macroscopic thermoelastic properties}

From tensile and compressive experiments (for details, see [8]), the Young's modulus is 7.1 GPa, and the Poisson's coefficient is 0.335 . From these values, the bulk modulus is $7.2 \mathrm{GPa}$ and the shear modulus is $2.7 \mathrm{GPa}$. From the thermal expansion experiments of Figs. 1 and 2. the volumetric thermal expansion modulus is close to $1.5 \times 10^{-4} \mathrm{~K}^{-1}$.

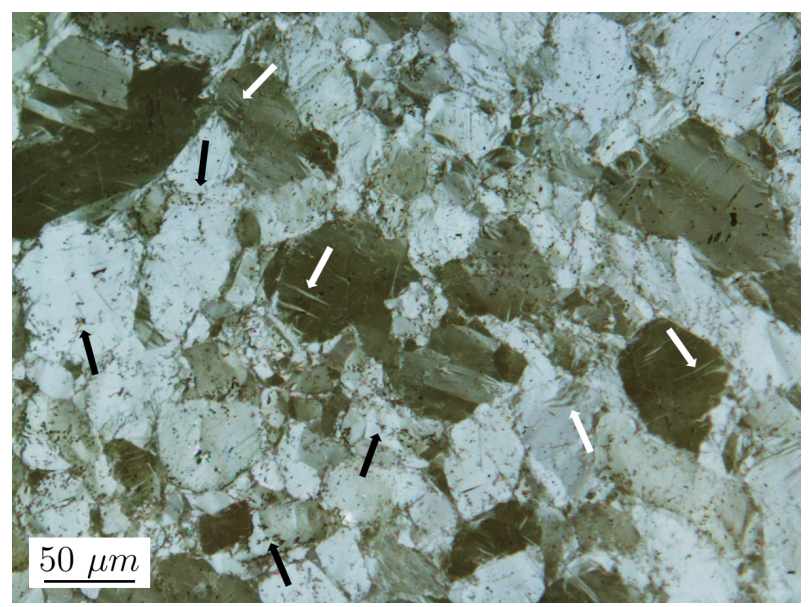

Figure 3: Optical microscopy, reflected polarized light. Black arrows: binder-filled intragranular porosity. White arrows: deformation bands. 


\section{Microstructure}

After pressing, the resulting solid, slightly porous, can be cut and polished by a standard metallographic-like technique. Fig. 3 was obtained by optical microscopy (reflected polarized non analyzed light). The TATB grains contain dark spots (black arrows), which are the remnant of the porosity of the initial powder, and were shown to be filled with binder. The grains also contain more or less straight bands (white arrows). Absent from the initial powder, these bands are thought to be the witness of the plastic deformation of TATB grains during pressing. The intergranular binder is not resolved.

In order to better reveal the polycrystalline nature of the explosive, SEM images are taken, after having etched the surface with a solvant of the binder. Fig. 4 clearly shows the grain boundaries, together with some intragranular microcracks (solid arrows). The etching process removes the binder from the intragranular porosity, which appears very clearly. In Fig. 4 , triple points (open arrows) can be seen as voids, contrarily to the optical micrograph of Fig. 3, taken approximately at the same magnification. This means that the etching process is also likely to have removed some of the smallest grains located at triple points, or even perhaps along grain boundaries. This should be kept in mind when evaluating grain size distributions.

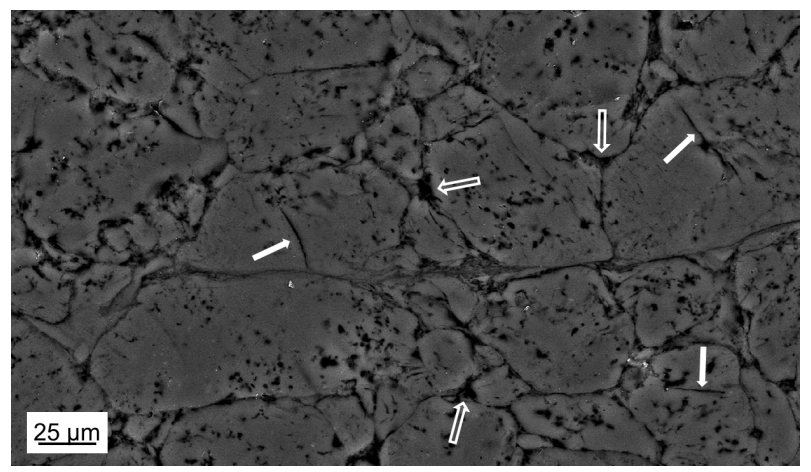

Figure 4: Scanning electron microscopy after etching. Solid arrows: microcracks. Open arrows: voids at triple points, probably induced by the etching process.

In what follows, binder-filled pores, intragranular microcracks and deformation bands will be discarded, to consider the polycrystalline character of the material only. For this purpose, a large mosaic of SEM images is taken, and segmented manually, by drawing a line of width 1 pixel around each grain. The result, with borders widened, is displayed in Fig. 5. The field is $5000^{2}$ pixels, and the resolution is $0.134 \mu \mathrm{m}$ per pixel. The manually segmented image is the main source of morphological data used in this work.

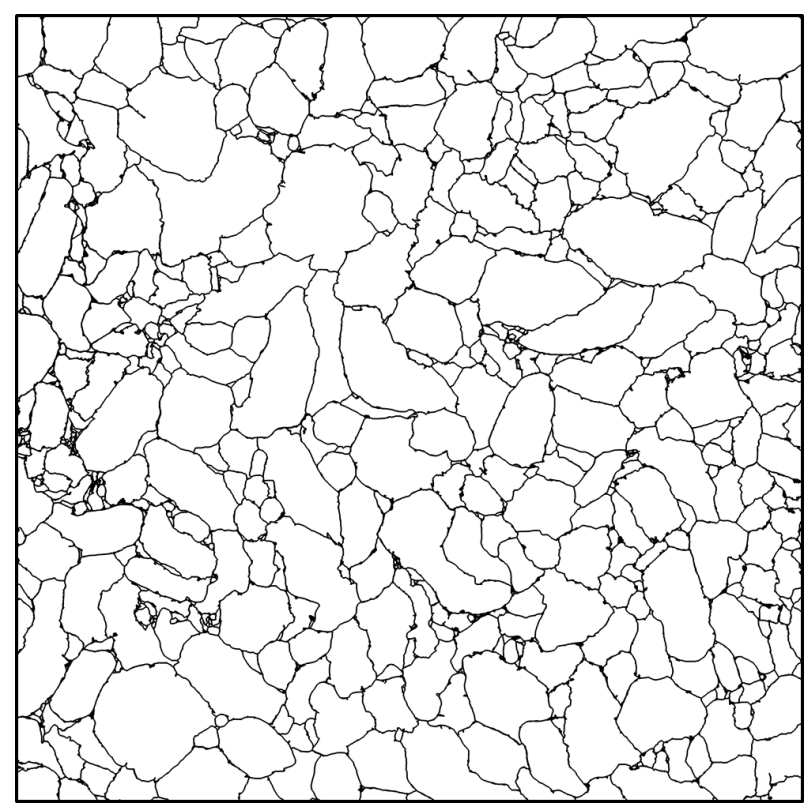

Figure 5: The polycrystalline microstructure after manual segmentation. Binder-filled porosity, deformation bands and microcracks have been removed.

Globally, the microstructure is isotropic (not shown, see [8]). Fig. 6] shows the distribution of grain areas $P(a)$, defined as the proportion of grains with given area $a$. It exhibits two peaks, centered at 1023 and 1,4 $\mu^{2}$ approximately, corresponding to equivalent disk radii of 18 and $0.67 \mu \mathrm{m}$ respectively. The grain size distribution measured by a succession of morphological openings [8] and the distribution of grain eccentricity (not shown) will be used in Section III. Also note that the grains are generally not convex, sometimes strongly, and have elongated shapes. 


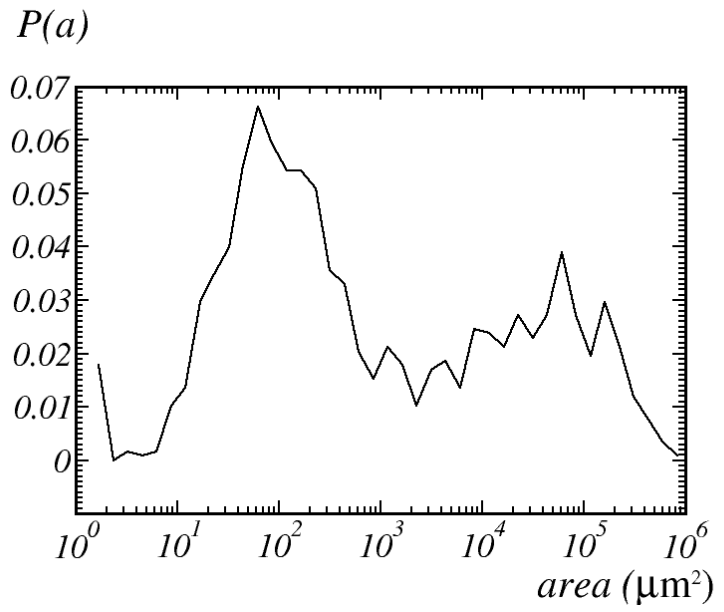

Figure 6: Distribution of grain areas.

\section{VIRTUAL MICROSTRUCTURE MODELING}

In this Section, a model of virtual microstructure is developed to mimic the real one and on which to perform thermomechanical simulations. This will be sought in the form of three-dimensional tessellations of space, by a nucleation and growth process. This approach is known to be sufficiently flexible to expect a reasonably realistic result (see for instance [12, 13, 14]), although the real material involves a completely different manufacturing process. The prototype of random tessellation models is the Poisson-Voronoï one, which involves simultaneous random nucleation and uniform growth. This model is not convenient for the present purpose [8], since it displays planar grain boundaries, far from the image of Fig. 5 .

For this reason, previous work [8] envisaged models derived from the so-called Johnson-Mehl one, which involves progressive nucleation and uniform growth. It was shown that a two-parameter accelerated nucleation model was necessary to mimic reasonably well the grain size distribution of the image of Fig. 5. The resulting model afforded curved grain boundaries, and therefore a certain non-convexity of grains. However, it did not reproduce their elongated character. In order to improve this aspect, a Johnson-Mehl-like model, involving progressive nucleation and uniform but anisotropic growth (see e.g. [13]), is implemented.
The nucleation process is assumed to follow a spacetime Poisson distribution. The spatial and temporal coordinates of grain nuclei are thus chosen at random in the interval $[0, L]^{3} \times[0, T]$ where $L$ is the spatial dimension of the simulated volume, and $T$ the duration of the nucleation process. The Poisson process is characterized by its intensity $\theta$, namely the (statistical) average of grain nuclei per unit volume and time $\left(\mu \mathrm{m}^{-3} \cdot \mathrm{s}^{-1}\right)$. Growth begins as soon as a new nucleus appears, and proceeds until adjacent grains meet, hence defining their boundaries. As a general rule, for Voronoï and Johnson-Mehl-like tessellations, a grain $G_{i}$ is defined as the influence zone of the $i$-th nucleus by:

$$
G_{i}=\left\{x ;\left|x-x_{i}\right|+v_{0} t_{i}<\left|x-x_{i}\right|+v_{0} t_{j} ; \quad i \neq j\right\}
$$

where $|$.$| is the distance function, x_{i}$ and $t_{i}$ (respectively $x_{j}$ and $t_{j}$ ) are the spatial and temporal coordinates of nucleus labeled $i$ (resp. $j$ ), and $v_{0}$ is the growth rate of the grains, assumed as uniform. In fact, the parameter $v_{0}$ acts as a spatio-temporal scaling parameter, and will be fixed arbitrarily as $v_{0}=1 \mu \mathrm{m} . \mathrm{s}^{-1}$ in the sequel. In order to introduce anisotropic growth [14], let the distance function be defined as follows:

$$
\begin{aligned}
|\mathbf{x}|= & \left\|\mathbf{x}-\mathbf{x}_{i}\right\|+\left(K^{2}-1\right) \\
& {\left[R_{1} \cos \phi \sin \psi+R_{2} \sin \phi \sin \psi+R_{3} \cos \psi\right] }
\end{aligned}
$$

where $\|$.$\| is the Euclidean distance, \theta$ and $\phi$ are the Euler angles of the local anisotropic principal direction, $K$ the anisotropy factor, and $R_{1}, R_{2}$ and $R_{3}$ are the components of the vector linking the point $\mathbf{x}$ to germ $\mathbf{x}_{i}$. This definition reduces to the Euclidean distance when $K=1$.

Therefore, the model is defined by the intensity $\theta$ of the nucleation process, by the anisotropy factor $K$, and by the distribution of anisotropy directions, which are taken uniformly on the unit sphere in the absence of precise knowledge. Those two parameters are sought in the following way. In previous work [8], the (isotropic growth) models were identified by matching the grain size distribution predicted by the model with that measured on the image of Fig. 5. In the present anisotropic case, the grain size distribution is complemented by a measurement of the elongation of the grains.

Those data are first measured on the segmented image (Fig. 5). Then, for each couple of parameters $\theta$ and 
$K$, several realizations of the virtual microstructure are generated. For each realization, a series of planar sections are extracted, used for the grain size and elongation measurements, and the results are averaged. The difference between predicted and experimental grain size and elongation data are then minimized by a simplex optimization algorithm [15]. The optimal couple of parameters is found to be $\theta=5 \times 10^{-7} \mu \mathrm{m}^{-3} \cdot \mathrm{m}^{-1}$ and $K=2.24$, and is illustrated in Fig. 7, to be compared with Fig. 5. This microstructure is discretized on a volume of $512^{3}$ voxels, and contains about 31,500 germs. See [16] for details on the anisotropic JohnsonMehl model and its optimization. The microstructure is periodized consistently with the boundary conditions applied in the FFT method, which is detailed hereafter.

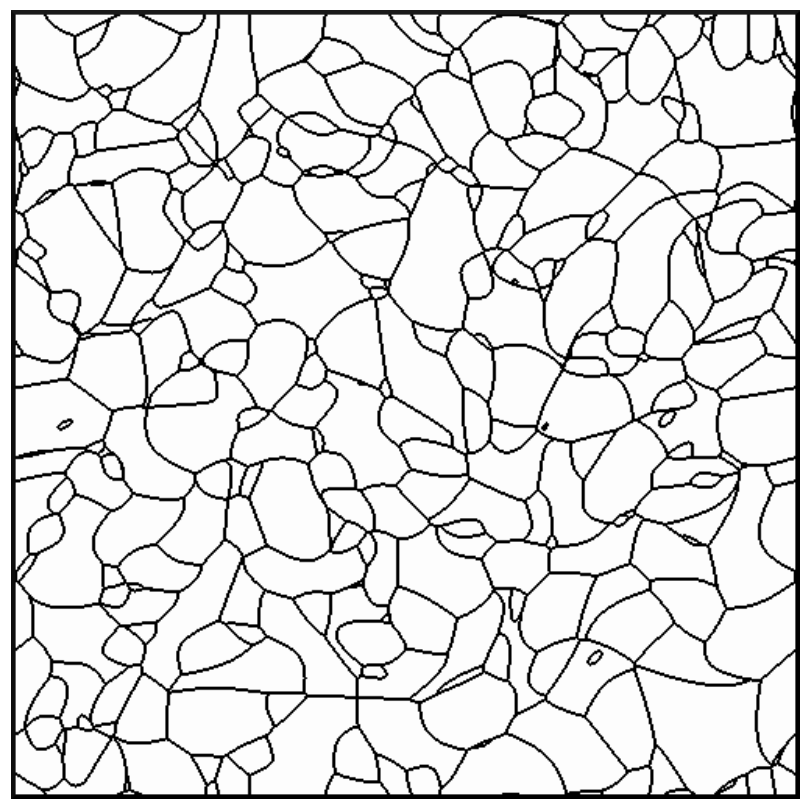

Figure 7: A planar slice of a realization of the optimum microstructure.

\section{Computations}

\section{Brief overview of the Fourier-based method}

The FFT-based computational method first proposed by Moulinec and Suquet [17] is chosen here, first because it needs no structured meshing and operates directly on voxels, and second because it is not demanding in computational resources. Besides, its accuracy has been shown to be comparable to that of the finite element method (see [18] for example). For room saving, the method will only be outlined here.

In brief, this full-field homogenization method deals with quasi-static problems under periodic boundary conditions, by seeking the solution in the form of Fourier series. The spatial average, non-fluctuating part of any field is considered as a macroscopic entity. Therefore, if macroscopic strains are prescribed, for instance, the method provides full stress and strain fields, and the spatial average of stresses is the macroscopic response to the prescribed strains. In the following, the so-called "accelerated scheme" FFT algorithm [19, 20] is used, together with the modified Green operator proposed in [21].

Although not being as mature, by far, as finite elements, the method is quite versatile, and has been used to solve electrical and thermal conductivity problems, as well as fluid flow through porous media, among others. Originally developed for elastic problems [17], it was recently adapted to thermoelasticity [8, 22], and works in the nonlinear field as well, including plasticity and even fracture (see for example [23, 24]).

\section{Microscopic thermoelastic properties of TATB}

As discussed above, the present work is restricted to thermoelasticity, and discards the binder, considering the grain boundaries as rigid with infinite strength. From [27, 28], it is known that the thermal expansion of TATB is reasonably linear between -50 and $100^{\circ} \mathrm{C}$. The dependence of elastic moduli on temperature is not known at present, but will be assumed negligible in the sequel.

Therefore, all is needed is the thermoelastic behavior of TATB. However, data are scarce in the literature. Concerning full anisotropic elastic constants of TATB, the only available reference to the authors' knowledge is the molecular dynamics work of Bedrov and coworkers [25]. They give the following values of the fully triclinic anisotropic elastic tensor in the Voigt 
notation (values given in GPa):

$$
\mathcal{C}=\left(\begin{array}{cccccc}
65.7 & 18.5 & 4.0 & -0.2 & -1.0 & 1.0 \\
& 62.0 & 5.0 & 0.6 & -0.5 & 1.0 \\
& & 18.3 & 0.2 & -0.4 & -0.4 \\
& & & 1.4 & 0.1 & 0.3 \\
& \text { sym } & & & 0.68 & 0.4 \\
& & & & & 21.6
\end{array}\right)
$$

The elastic tensor is expressed here in the $\left(\mathbf{e}_{1}, \mathbf{e}_{2}, \mathbf{e}_{3}\right)$ coordinate system for which $\mathbf{e}_{1}$ is parallel to the a direction of the crystal, $\mathbf{e}_{2}$ lies in the $(\mathbf{a}, \mathbf{b})$ plane, and $\mathbf{e}_{3}$ is chosen so as to form a direct orthonormal basis. Valenzano and co-workers [26] provide partial values of the elastic tensor ot TATB, derived by an analogous method. Their values are somewhat $20 \%$ higher than those of Bedrov and co-workers.

Concerning the thermal expansion tensor, two sources are available, giving quite different values. Kolb and co-authors [27] perform X-ray diffraction experiments on TATB single crystals between -50 and $140^{\circ} \mathrm{C}$, and follow the evolution of the six crystal cell parameters with temperature. From these data, it is possible to compute the components of the (linear) thermal expansion tensor (see the appendix of ref. [8]) in the same coordinate system as above, expressed here in normal tensorial notations (values given in $10^{-6} \mathrm{~K}^{-1}$ ) :

$$
\alpha=\left(\begin{array}{ccc}
8.28 & 1.67 & 13.07 \\
& 29.04 & 0.2 \\
\text { sym } & & 264.7
\end{array}\right)
$$

Sun and co-workers [28] provide the same data, but seemingly using powder diffraction, between 20 and $240^{\circ} \mathrm{C}$. They find the quite different following results (same units, same coordinate system):

$$
\alpha=\left(\begin{array}{ccc}
12.29 & -0.95 & 19.41 \\
& 9.022 & -37.39 \\
\text { sym } & & 166.2
\end{array}\right)
$$

\section{Predicted overall properties}

Calculations are performed on three different virtual microstructures, namely Voronoï, Johnson-Mehl and the above-described anisotropic Johnson-Mehl tessellations. For every grain of each microstructure, the orientations of the anisotropic axes are chosen at random, and the elastic and thermal expansion tensors are rotated with respect to the laboratory frame. This is done with the elastic data of Bedrov and co-workers [25], and with both the thermal expansion data of Kolb and co-workers [27] and of Sun and co-workers [28]. Available data being more than scarce in the literature about the correlation between crystallographic and morphological grain preferred directions, we choose here to keep them uncorrelated.

If a calculation is done by applying $\bar{\varepsilon}_{11}=1$, $\bar{\varepsilon}_{12}=\bar{\varepsilon}_{22}=\bar{\varepsilon}_{13}=\bar{\varepsilon}_{23}=\bar{\varepsilon}_{33}=0$, and $\Delta \bar{T}=0$ to the volume, where the overbars denote macroscopic quantities, then $\bar{C}_{11}=\bar{\sigma}_{11}, \bar{C}_{12}=\bar{\sigma}_{12}$, and so on. Therefore, the components of the elastic and thermal expansion overall tensors can be obtained by performing seven suitably chosen calculations. The apparent elastic tensor is symmetric at convergence of the FFT algorithm and nearly isotropic for large-enough volume sizes. For instance, for the anisotropic, $512^{3}$-voxels Johnson-Mehl model, all elastic components $\bar{C}_{i j}$ differ from less than $0.8 \%$ from that of an isotropic elastic tensor. The macroscopic bulk, shear and volumetric thermal expansion moduli are derived and listed in Table 1

\begin{tabular}{|c|c|c|c|c|}
\hline & $K$ & $\mu$ & \multicolumn{2}{|c|}{$\alpha\left(10^{-4} \mathrm{~K}^{-1}\right)$} \\
\cline { 4 - 5 } microstructure & $(\mathrm{GPa})$ & $(\mathrm{GPa})$ & $\mathrm{Kolb}$ & Sun \\
\hline Voronoï & 17.24 & 6.72 & 2.33 & 1.38 \\
Johnson-Mehl & 17.03 & 6.92 & 2.53 & 1.42 \\
anisotropic & 17.08 & 6.69 & 2.44 & 1.39 \\
\hline experimental & 7.1 & 2.7 & \multicolumn{2}{|c|}{1.5} \\
\hline
\end{tabular}

Table 1: Predicted macroscopic thermoelastic properties vs. eperimental data. FFT results on the TATB volumetric thermal expansion $\alpha$ are computed using data from either Kolb et al. 27] or Sun et al. [28] for the monocrystal.

The results are almost insensitive to the details of the microstructure, at least at constant granulometry, as usually observed for linear behavior. The bulk and shear moduli show little variations but, not surprisingly, the volumetric thermal expansion modulus appears very sensitive to the thermal expansion input data. It is difficult to compare the present predictions to experimental data, because the binder has been neglected in the present calculations. However, it was 
observed in previous work [8] that including the effect of binder induced strong variations of the bulk and shear moduli, but had little effect on the thermal expansion modulus. This is consistent with the present findings, since a rather good match is observed between the present predictions involving the data of Sun and co-workers [28] and experimental findings (see Figs. 1] and 2).

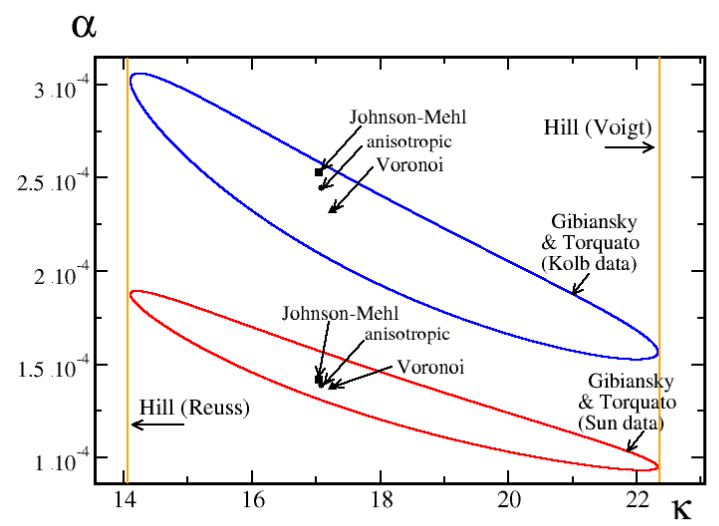

Figure 8: Predicted volumetric thermal expansion modulus vs the theoretical bounds of Gibiansky and Torquato [29]. The calculations involving the input data of Kolb and coworkers [27] are given in blue, and those involving the input data of Sun and co-workers [28] are given in red.

As a partial verification of the validity of the computational method, the present results are compared to existing theoretical bounds. For anisotropic polycrystals, Gibiansky and Torquato [29] provide an upper and a lower bound to the volumetric thermal expansion modulus as a function of the bulk modulus. The results of Table 1 are compared to those bounds in Fig. 8 , which shows the computational results to lie within the bounds. In this figure, the two vertical lines are the Hill-Reuss bound, obtained for uniform applied strain, and the Hill-Voigt one, obtained for uniform applied stress.

Although not being a definite validation of the computational method, this lends some confidence to the results. Besides, note the general trend indicated by the bounds. The thermal expansion modulus decreases as the bulk modulus increases, hence illustrating the growth of internal stresses and their effect on thermal expansion.

\section{Representative volume element}

Since calculations are necessarily performed on finite size volumes, it is legitimate to examine the issue of representativeness. The computation volume can be said to be representative when no statistical fluctuation of overall properties are recorded, in other words when the volume is sufficiently large and ergodicity is reached. Strictly speaking, this can only be the case for infinite systems. Therefore, following Kanit and co-workers [30], the volume will be said to be representative when statistical fluctuations, i.e. variance of the overall property of interest will stay below a given threshold. Observe that there is a priori no reason why the size of the representative volume element (RVE) should be identical for different properties, and that morphological and mechanical RVE are distinct concepts. In practice, this is done in the following way [8]. A single set of seven computations are performed on one large volume, here $512^{3}$ voxels, providing a three-dimensional chart of the thermomechanical fields used to derive the selected property. This chart is then divided into eight non overlapping sub-volumes, on which the selected property can again be determined. The variance of this property can be estimated from the eight sub-volumes results. The procedure is performed again on sub-subvolumes, and so on until voxel sized volumes are reached. In this context, any property of interest $Z$, here the bulk, shear or thermal expansion modulus, can be considered as a random variable. Then, it can be shown [31] that when $V \gg A_{3}^{Z}$, the following rule applies:

$$
\frac{D_{Z}^{2}(V)}{D_{Z}^{2}}=\frac{A_{3}^{Z}}{V}
$$

where $D_{Z}^{2}(V)$ is the (volume dependent) variance of $Z$, $D_{Z}^{2}$ its point variance. Eqn. 3 defines the integral range $A_{3}^{Z}$. Then, following [8, 30], the size of the RVE is given by:

$$
V_{R V E}=\frac{4 D_{Z}^{2} A_{3}^{Z}}{\eta^{2} Z^{2}}
$$


where $\eta$ is the selected relative precision on $Z$.

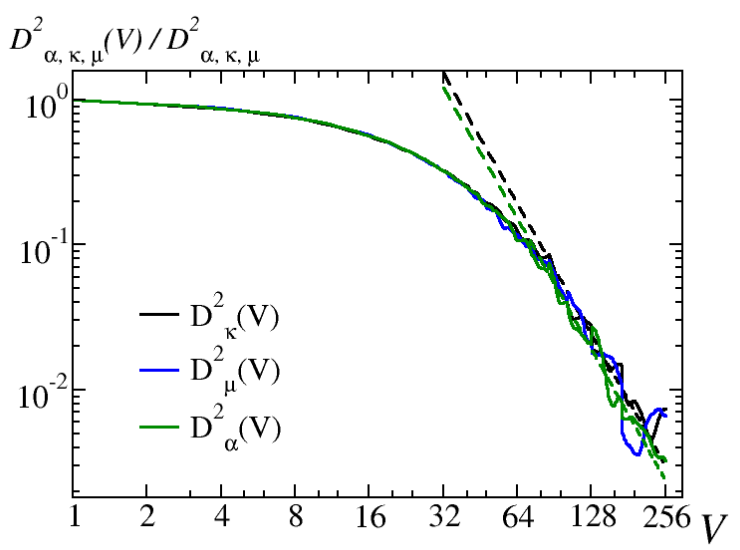

Figure 9: Variances $D_{\alpha, \kappa, \mu}^{2}(V)$ of the apparent thermal and elastic moduli as a function of the volume size $V$. Dashed-lines: fits $D_{\alpha, \kappa, \mu}^{2}(V) \sim 1 / V$ for large $V$ (see 4 . The green and blue dashed lines almost coincide.

The integral range, point variances and relative precision are computed using various stress fields and appropriate macroscopic strain and thermal loading. For the bulk modulus $\kappa$, we use the field $\sigma_{m}(\mathbf{x})=\frac{1}{3} \sigma_{k k}(\mathbf{x})$ with applied strain loading $\bar{\varepsilon}_{11}=1$ and $\bar{\varepsilon}_{i j}=0$ for $(i, j) \neq(1,1)$. For the shear modulus $\mu$, use is made of the field $\sigma_{12}(\mathbf{x})$ with applied strain loading $\bar{\varepsilon}_{12}=\bar{\varepsilon}_{21}=1$ and other components set to zero. For the thermal expansion coefficient, we use the field $\sigma_{m}(\mathbf{x})$ with thermal loading $\Delta T=1$ and strain loading $\bar{\varepsilon}_{i j}=0$. Calculations are performed on a $512^{3}$ voxels anisotropic Johnson-Mehl microstructure made of about 3,000 grains, with local thermal expansion tensor given by [27]. The variances $D_{\alpha, \kappa, \mu}^{2}(V)$ are represented in Fig. (9) for increasing subvolume size $V$. This provides the respective integral ranges of $A_{3}^{K}=37^{3}$ voxels $\left(48^{3} \mu \mathrm{m}^{3}\right), A_{3}^{\mu}=34^{3}$ voxels $\left(44^{3} \mu \mathrm{m}^{3}\right)$, and $A_{3}^{\alpha}=34^{3}$ voxels $\left(45^{3}{\mu m^{3}}^{3}\right)$ for the bulk, shear and thermal expansion moduli respectively. This corresponds, for this volume size, to respective relative precisions of $1.1,1.8$ and $1.4 \%$. Conversely, a volume containing approximately 360,000 grains would be necessary to compute the bulk modulus with a relative precision of $0.1 \%$. The slightly weaker integral range observed for the shear modulus is probably the result of a relatively low contrast between the shear moduli of grains showing different crystallographic orientations, which induces fluctuations of fields at a small scale only.

\section{Local fields}

Up to now, only the overall properties have been studied. Fig. 10 displays an example of the pressure field if the volume is submitted to a temperature increase of $1 \mathrm{~K}$ and zero strains. Since the local behavior is linear, the pressure can also be read as pressure unit per $\mathrm{K}$. This pressure map illustrates the strongly heterogenous nature of the field, especially across grain boundaries. This is expected to become crucial when nonlinear processes will be at stake.

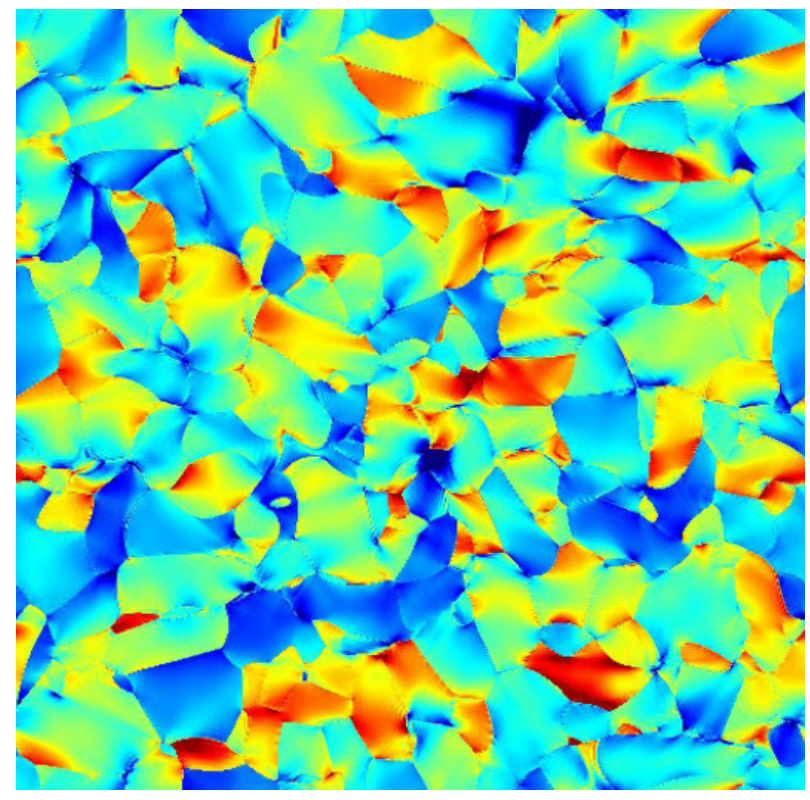

Figure 10: Example of pressure field under applied temperature increase of $1 \mathrm{~K}$ and zero strains. Color scale: minimum (red) is $-5.1 \mathrm{MPa}$ or MPa. $\mathrm{K}^{-1}$, maximum (blue) is -3.6 MPa or MPa.K $K^{-1}$,

In order to get a more precise overview of the heterogeneity of the fields, Fig. 11 shows the strain histograms corresponding to the same calculation as above. It has been checked that the latter are nearly 
identical at 2-times smaller discretization (not shown). The ordinate axis indicates the normalized probability (or equivalently the normalized number of voxels) of finding a strain value of $\varepsilon_{i j}$. As expected, the shear strains are centered around a zero value; $\varepsilon_{\mathrm{m}}$ is the mean strain and $\varepsilon_{\text {eq }}$ is the von Mises equivalent strain.

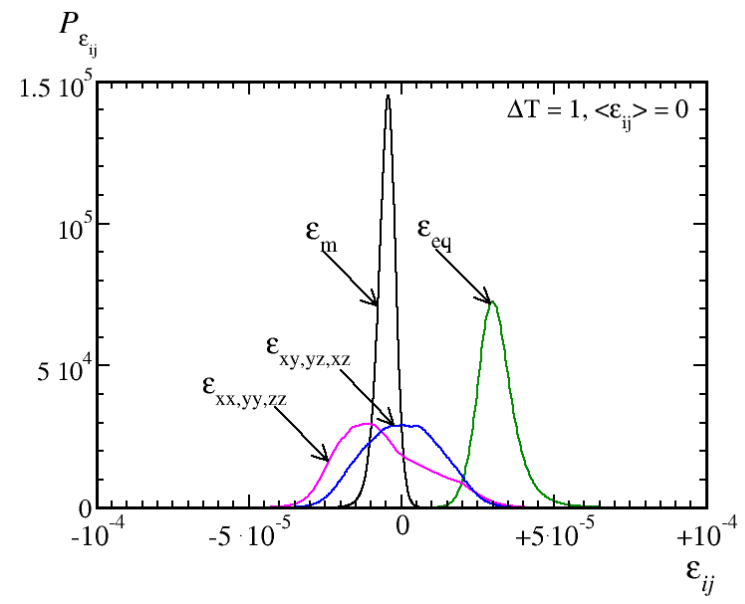

Figure 11: Strain histograms for the same calculation as in Fig. 10

FFT results indicate that the field histograms for the strain and stress components are almost identical for the Voronoï, standard and anisotropic Johnson-Mehl models (not shown). This lack of sensitivity with respect to the microstructure has been noticed in other works in linear elasticity [32]. In the present material, this is explained by the moderate contrast between elastic moduli. For instance, the Young's moduli in the inplane and out-of-plane directions are about 64 and 18 GPa. Stronger effect of the microstructure is expected in highly nonlinear regimes.

\section{CONCLUSION AND FUTURE WORK}

The work presented here constitutes the second step of the development of a numerical homogenization tool dedicated to a class of TATB-based polymer-bonded explosives. It involves an improved representation of the microstructure, and can already deliver some thermoelastic predictions. The predicted volumetric thermal expansion coefficient is close to experimen- tal data whereas the elastic moduli are significantly overpredicted. However, it suffers from neglecting the presence of thin layers of intergranular polymeric binder, already shown to have pronounced effects on the macroscopic elastic properties of the explosive. Accounting for this binder is clearly the next step of the development. Numerical results indicate that the presence of a soft interphase located inbetween crystals significantly reduces the elastic moduli. Surprisingly, the thermal expansion modulus is apparently not affected [7, 8]. This effect is not clearly understood, and requires a dedicated study. Nevertheless, additional work is required to account for the binder. The latter is a key constituent, as it controls partly the levels of stresses imposed to the TATB grains as well as the grain boundaries. The role of a weakened interphase is equally important in nonlinear regimes (see e.g. [33]). A sensible investigation into sub-macroscopic scale physical processes cannot be undertaken unless the local role of the binder is accurately understood and described.

Acknowledgments. This work was performed with the financial support of the French Commissariat à l'Énergie Atomique. The authors are indebted to JeanLuc Brigolle and Didier Picart, who provided macroscopic data, to Philippe Lambert, who performed the manual segmentation of experimental images, and to Mathieu Faessel, who helped for the morphological measurements, and for many fruitful discussions.

\section{REFERENCES}

[1] W. R. Blumenthal, G. T. Gray, D. J. Idar, M. D. Holmes, P. D. Scott, C. M. Cady, D. D. Cannon, Shock Compression of Condensed Matter (1999), Snowbird (UT), USA.

[2] D. G. Thompson, R. Deluca, G. B. Brown, J. Energetic Mat. (2012), 30:4, 299-323.

[3] H. F. Rizzo, J. R. Humphrey, J. R. Kolb, Propellants, Explosives, Pyrotechnics (1981), 6, 27-36.

[4] J. L. Maienschein, F. Garcia, Thermochimica Acta (2002), 384, 71-83. 
[5] R. Belmas, L. Gautier, C. David, D. Picart, C. Le Gallic, P. Lambert, Propellants, Explosives, Pyrotechnics (2005), 30:2, 101-104.

[6] A. Maiti, R. H. Gee, D. M. Hoffman, L. E. Fried, J. Appl. Phys. (2008), 103, 053504.

[7] A. Ambos, H. Trumel, F. Willot, D. Jeulin, M. Biessy, $15^{\text {th }}$ Int. Symp. on Detonation (2014), San Francisco (CA), USA.

[8] A. Ambos, F. Willot, D. Jeulin, M. Faessel, B. Figliuzzi, H. Trumel, Int. J. Solids Struct. (2015), 60-61, 125-139.

[9] D. G. Thompson, R. Deluca, S. Hagelberg, 28th Compatibil ity, Aging and Stockpile Stewardship Conference (2009), Albuquerque (NM), USA.

[10] P. C. Souers, P. Lewis, M. Hoffman, B. Cunningham, Propellants, Explosives, Pyrotechnics (2011), 36, 335-340.

[11] J. Sun, B. Kang, H. Zhang, Y. Liu, Y. Xia, Y. Yao, X. Liu, Central Eur. J. Energetic Mat. (2011), 8 (1), 69-79.

[12] J. Moller, Adv. Appl. Probab. (1992), 24, 814-844.

[13] H. Altendorf, F. Latourte, D. Jeulin, M. Faessel, L. Saintoyant, Image An. \& Ster. (2014), 33 (2), 121-130.

[14] D. Jeulin, Lecture Notes in comput. Sci. (2013), $7883,25-36$.

[15] J. A. Nelder, R. Mead, The Computer J. (1965), 7 (4), 308-313.

[16] J.-B. Gasnier, B. Figliuzzi, F. Willot, D. Jeulin, M. Faessel, H. Trumel, in preparation.

[17] H. Moulinec, P. Suquet, C. R. Acad. Sci. Paris II (1994), 318, 1417-1423.

[18] A. Prakash, R. A. Lebensohn, Modelling Simul. Mater. Sci. Eng. (2009), 17, 064010.
[19] D. J. Eyre, G. W. Milton, Eur. Phys. J. Appl. Phys. (1999), 6 (1), 41-47.

[20] V. Vinogradov, G. W. Milton, Int. J. for Num. Meth. Engng (2008), 76 (11), 1678-1695.

[21] F. Willot, C. R. Acad. Sci. Mécanique (2015), 343 (3), 232-245.

[22] B. S. Anglin, R. A. Lebensohn, A. D. Rollett, Comput. Mat. Sci. (2014), 87, 209-217.

[23] R. A. Lebensohn, A. D. Rollett, P. Suquet, JOM (2011), 63 (3), 13-18.

[24] J. Li, X. X. Tian, R. Abdelmoula, Int. J. Fract. (2012), 173, 135-146.

[25] D. Bedrov, O. Borodin, G. D. Smith, T. D. Sewell, D. M. Dattelbaum, L. L. Stevens, J. Chem. Phys. (2009), 131, 224703.

[26] L. Valenzano, W. J. Slough, W. F. Perger, Shock Compression of Condensed Matter (2011), Chicago (IL), USA.

[27] J. R. Kolb, H. F. Rizzo, Propellants, Explosives, Pyrotechnics (1979), 4, 10-16.

[28] J. Sun, B. Kang, C. Xue, Y. Liu, Y. Xia, X. Liu, W. Zhang, J. Energetic Mat. (2010), 28 (3), 189-201.

[29] L. V. Gibiansky, S. Torquato, J. Mech. Phys. Solids (1997), 45 (7),1223-1252.

[30] T. Kanit, S. Forest, I. Galliet, V. Monoury, D. Jeulin, Int. J. Solids Struct. (2003), 40, 3647-3679.

[31] C. Lantuéjoul, J. Microsc. (1991), 161, 387-403.

[32] M. Sauzay, J. Liu, F. Rachdi, L. Signor, T. Ghidossi, P. Villechaise, Adv. Mat. Res. (2014), 891-892, 833-839.

[33] L. Delannay, P. Yan, J.F.B. Payne, N. Tzelepi, Comput. Mat. Sc. (2014) 87, 129-137. 